\title{
Les facteurs influençant les indicateurs de performance à l'URENI de la pédiatrie du CHU Gabriel Touré
}

\section{Factors influencing performance indicators at pediatrics' URENI of teaching hospital Gabriel Touré}

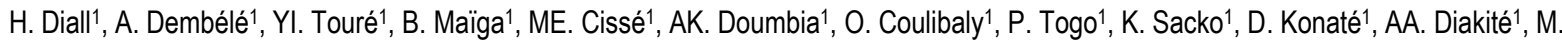

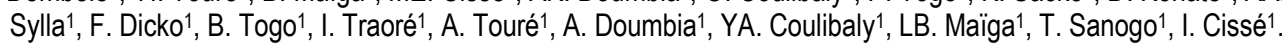

1 Département de pédiatrie du CHU Gabriel Touré

Auteur correspondant : Dr Adama DEMBELE, Pédiatre, EnseignantChercheur, Département de pédiatrie, CHU Gabriel Touré, Bamako, adamadembele2206dochgt@yahoo.fr, Mali.

\section{Résumé :}

Introduction: Au Mali, les unités de récupération et d'éducation nutritionnelle intensive ont été créées partout sur le territoire et celle de la pédiatrie du CHU Gabriel TOURE a commencé ses activités en Mai 2010. L'objectif de notre étude était d'analyser les facteurs influençant les indicateurs de performance chez les enfants de moins de 5 ans à l'URENI de la Pédiatrie du CHU Gabriel TOURE. Matériel et Méthodes: Etude transversale, descriptive et analytique, elle s'est déroulée sur une période de 6 mois (du 01 juin au 30 novembre 2016). Tous les enfants malnutris sévères de moins de 5 ans hospitalisés ont été inclus. Résultats : Pendant la période d'étude, 4724 enfants ont été hospitalisés dans le service, 259 cas de malnutrition aigüe sévère soit $5,5 \%$. La tranche d'âge $12-23$ mois était la plus représentée avec $44,79 \%$. Le sex-ratio était de 0,90 . Les patients étaient exclusivement allaités au sein $(76,06 \%)$ avant l'âge de 6 mois et $36,68 \%$ n'avaient pas bénéficié d'aliments de supplément après 6 mois. Le motif de consultation le plus fréquent était l'anorexie $(66,79 \%)$. Le marasme était la forme de malnutrition la plus représentée (87,65\%). L'échec au test de l'appétit était la principale complication de la prise en charge avec $42,86 \%$ des cas Les traités avec succès étaient de $54,83 \%$. Nous avons enregistré 18,53\% d'abandon de traitement. II y'avait une association statistiquement significative entre les traités avec succès et la profession du père $(O R=2,7 ; p=0,028)$. L'association entre le décès et les complications était statistiquement significative $(O R=21,345 ; p=0,001)$. II y'avait une association statistiquement significative entre l'abandon du traitement et les problèmes financiers $(O R=3,037 ; p$ $=0,005)$. Le taux de décès était de 23,55\%. Conclusion : Les problèmes financiers des parents et l'association des complications (pathologies graves) constituent des facteurs qui influencent considérablement sur les indicateurs de performance.

Mots clés : Indicateurs, Performance, URENI, Enfants (moins de 5 ans), Bamako.

\footnotetext{
Abstract:

Introduction: In Mali, Intensive nutritional recovery and education units have been established throughout the country and the pediatric unit of Gabriel Touré Teaching Hospital started in May 2010. The objective was to analyze the factors influencing the performance
}

indicators at the Intensive nutritional recovery unit in the Pediatric of Gabriel Touré Teaching Hospital. Material and Methods: Cross-sectional, descriptive and analytical study, it took place over a period of 6 months (June 1 to November 30, 2016). All severe malnourished children under 5 years of age were included. The parents or caregivers of these children as well as the staff of the Intensive nutritional recovery unit were asked about their opinions. Result: During the study period, 4724 children were hospitalized in the service. There were 259 cases of severe acute malnutrition with a frequency of $5.5 \%$. The 12-23 month age group was the most represented with $44.79 \%$. Sex ratio of 0.90 . Patients were exclusively breastfed (76.06\%) before 6 months of age and $36.68 \%$ had not benefited from supplement foods after 6 months. The most common reason for consultation was anorexia with $66.79 \%$ of cases. Muscle wasting was the most found sign on physical examination (66.79\%). Marasmus was the most represented form of malnutrition (87.65\%). The failure of the appetite test was the main complication of management with $42.86 \%$ of cases. The combination of milk F75 + F100 + PPN was used for management in $87.26 \%$ of cases. Treated successfully are $54.83 \%$. We recorded $18.53 \%$ dropout. There was a statistically significant association between the treaties successfully and the father's occupation (OR $=2.7, p=0.028)$. The association between death and complications was statistically significant $(O R=21.345, p=0.001)$. There was a statistically significant association between treatment discontinuation and financial problems (OR = $3.037, p=0.005)$. The death rate was $23.55 \%$. Conclusion: The financial problems of the parents and the association of the complications (serious pathologies) constitute factors which influence considerably on the indicators of performance.

Keywords: Indicators, Performance, INRU, Children (under 5 years), Bamako.

\section{Introduction}

La malnutrition est un ensemble de manifestations dues à un apport inadéquat en quantité et / ou en qualité dans l'alimentation de substances nutritives nécessaires à la croissance normale et au bon fonctionnement de l'organisme, que cet ensemble se manifeste cliniquement ou ne soit décelable que par des analyses biochimiques, anthropométriques, ou physiologiques [1].

La sous nutrition est à l'origine de $45 \%$ des décès chez les enfants de moins de 5 ans, représentant plus de 3 millions de décès par an [2].

Le retard de croissance intra-utérin et l'allaitement nonoptimal causent ensemble plus de 1,3 million de décès, 
ou $19,4 \%$ de la totalité des décès d'enfants de moins de 5 ans, soit $43,5 \%$ de tous les décès liés à la nutrition [2]. Le risque de décès est quatre fois plus élevé pour un enfant atteint d'un retard de croissance grave et neuf fois plus élevé pour un enfant souffrant d'émaciation grave [3].

L'émaciation modérée et grave représentent une forme aiguë de sous-nutrition et les enfants qui en souffrent sont confrontés à un risque nettement accru de décès. Au niveau mondial, la prévalence de la maigreur s'élevait à $8 \%$ en 2011 , touchant ainsi 52 millions d'enfants de moins de 5 ans (soit une baisse de 11\% par rapport aux 58 millions d'enfants estimés en 1990) [2]. La prévalence de la maigreur sévère atteignait quant à elle 2,9 \%, affectant 19 millions d'enfants. A l'échelle mondiale, 70\% des enfants atteints de maigreur habitent en Asie, plus particulièrement en Asie du Sud où $15 \%$ des enfants sont touchés (28 millions) [2].

En Afrique subsaharienne, près d'un enfant de moins de 5 ans sur dix soit $9 \%$, a souffert d'émaciation en 2011 , une prévalence qui a diminué d'environ $10 \%$ depuis 1990. Cependant, en raison de la croissance de la population, la région abrite aujourd'hui un tiers d'enfants émaciés en plus par rapport à 1990. La proportion du nombre d'enfants touchés en Afrique subsaharienne a augmenté par rapport au chiffre total mondial, au cours de la même période [4].

Au Mali, selon les résultats de l'enquête SMART 2015, la prévalence de la malnutrition aigüe globale est de 12,4\%. D'après les résultats par région, la situation nutritionnelle est jugée précaire dans toutes les régions enquêtées avec des prévalences situées entre $10 \%$ et $15 \%$, sauf la région de Tombouctou dont la prévalence de la malnutrition aigüe globale $17,5 \%$ dépasse le seuil critique de $15 \%$. Les petits enfants âgés de 6 à 23 mois sont plus affectés par la malnutrition aiguë que ceux âgés de 24 à 59 mois. Pour la malnutrition chronique (retard de croissance) et l'insuffisance pondérale, les prévalences nationales sont respectivement de $29,3 \%$ et de $24,2 \%$ [5].

Le Mali à l'instar des autres pays de l'Afrique a adopté une stratégie intégrée de prise en charge de la malnutrition aiguë [6]. Un protocole national de prise en charge de la malnutrition aigüe a été élaboré et des unités de récupérations nutritionnelles ont été créées sur toute l'étendue du territoire. L'unité de récupération et d'éducation nutritionnelle intensive de la pédiatrie de l'hôpital de Gabriel TOURE a commencé ses activités en Mai 2010 et reçoit tous les enfants malnutris hospitalisés dans le département de pédiatrie. La prise en charge de la malnutrition aigüe sévère est évaluée par les indicateurs de performance dont le taux de traités avec succès, le taux de décès, le taux d'abandon, le taux de non-répondant et la durée de séjour.

Au cours de l'année 2015, l'URENI de l'hôpital Gabriel Touré a enregistré selon le rapport de la Division Nutrition de la Direction Nationale de la Santé, 564 admissions dont 375 cas traités avec succès, 53 cas de décès, 130 cas d'abandon soit respectivement: $67 \%, 10 \%$ et $23 \%$. Ces statistiques sont en deçà de celles attendues dans le protocole PCIMA qui doivent être de plus de $75 \%$ et de moins de $10 \%$ et $15 \%$ respectivement. Elles sont certes en dessous des valeurs alarmantes mais restent très préoccupantes particulièrement pour le taux d'abandon. Ainsi une analyse approfondie des facteurs s'avère nécessaire afin d'identifier les raisons qui expliquent ces faibles niveaux des indicateurs de performance. D'où la nécessité de mener une étude à l'URENI pour comprendre son fonctionnement et sa capacité à répondre aux besoins des malnutris.

L'objectif de notre étude était d'analyser les facteurs influençant les indicateurs de performance à l'URENI chez les enfants de moins de 5 ans dans le Département de Pédiatrie du CHU Gabriel Touré pour améliorer la qualité de la prise en charge.

\section{Matériel et Méthodes CADRE ET LIEU D'ETUDE}

Notre étude s'est déroulée dans le département de pédiatrie du CHU - Gabriel Touré de Bamako. Situé au centre de la ville, ce département reçoit les patients de toutes les communes de Bamako et ceux référés par les autres localités du Mali. Malgré l'existence des centres de santé communautaires et les centres de santé de référence, l'affluence y reste encore très élevée. II est composé d'un service de pédiatrie générale, d'un service des urgences pédiatriques ;

- un service de néonatologie auquel est rattachée I'URENI (Unité de Récupération et d'Education Nutritionnelle Intensive).

L'URENI assure la prise en charge de la malnutrition aigüe sévère avec complication conformément aux recommandations du protocole national. Son personnel comprend un médecin Pédiatre, un technicien supérieur de santé, une technicienne de santé, et une aidesoignante.

\section{TYPE d'étude et critères d'inclusion}

II s'agissait d'une étude transversale, descriptive et analytique. Elle s'est déroulée sur une période de 6 mois allant du 01 juin au 30 novembre 2016. Nous avons inclus tous les enfants malnutris aigues sévères de moins de 5 ans hospitalisés. Nous avons utilisé une approche quantitative pour estimer les paramètres relatifs aux enfants malnutris hospitalisés. La collecte des données concernant les enfants hospitalisés a été faite à travers l'exploitation des dossiers d'hospitalisation et des fiches thérapeutiques de suivi individuel des enfants à I'URENI.

Les données ont été saisies et analysées par les logiciels Epi info 7, SPSS 20 et STATA 10. Pour l'analyse univariée, les variables qualitatives ont été présentes par les mesures de la fréquence. Quant aux variables quantitatives la description a été faite par le calcul de la moyenne, l'écart-type et de l'intervalle de confiance à $95 \%$. 
Pour l'analyse bivariée et multi variée, nous avons fait un modèle de régression logistique.

Le seuil de signification statistique retenu est fixé à $5 \%$. Dans le cadre du respect de la confidentialité et de la dignité de la personne humaine, le consentement verbal éclairé et libre des parents ou accompagnants a été obtenu avant la collecte après une explication détaillée sur les objectifs de l'étude et les procédures de collecte de données.

\section{Résultats}

Pendant notre période d'étude, 4724 enfants ont été hospitalisés à la pédiatrie de l'hôpital Gabriel Touré, dont 259 cas de malnutrition aigüe sévère $5,5 \%$. La tranche d'âge 12-23 mois était la plus représentée avec 44,79\%. Les hommes représentaient $47,49 \%$ avec un sex-ratio de 0,90 . Les patients étaient allaités exclusivement au sein avant 6 mois dans 76,06 \% des cas. Environ 36,68\% des enfants après 6 mois n'avaient jamais bénéficié d'aliments de supplément. La majorité des pères des patients $(79,54 \%)$ n'étaient pas instruits et beaucoup $(81,08 \%)$ travaillaient dans le secteur informel. Les mamans de nos patients n'étaient pas instruites dans $79,54 \%$ des cas et $78,76 \%$ étaient femmes au foyer. Les motifs de consultation fréquemment évoqués étaient l'anorexie avec $66,79 \%$ suivie de la toux $66,41 \%$.

La fonte musculaire était le signe physique le plus présent avec $66,79 \%$ des cas suivie des candidoses 66,41 . Le marasme était la forme de malnutrition la plus représentée avec $87,65 \%$ des cas. Parmi les complications rencontrées à l'admission des patients, l'échec au test de l'appétit était la plus fréquente avec $42,86 \%$ des cas. La durée moyenne de séjour était inférieure à quatre semaines dans $94,98 \%$ des cas ; plus de la moitié des enfants malnutris soit $54,83 \%$ ont été traités avec succès ; $23,55 \%$ sont décédés et $18,53 \%$ ont abandonné le traitement. L'analyse des facteurs a porté essentiellement sur les principaux indicateurs de performance : "traités avec succès », "les décès » et les «abandons» qui sont considérées comme les variables dépendantes lors de la régression logistique. L'ensemble des facteurs influençant ces indicateurs de performance à l'URENI (ou variables explicatives) ont été analysés. II y'avait une association statistiquement significative entre les traités avec succès et la profession salariale du père $(O R=2,7 ; p=0,028)$.

L'association entre le décès et les complications était statistiquement significative $(O R=21,345 ; p=0,001)$. ॥ $y$ avait une association statistiquement significative entre l'abandon et les problèmes financiers $(O R=3,037 ; p=$ 0,005) (Tableau XII)

\section{DISCUSSION}

$\mathrm{Au}$ cours de notre période d'étude, nous avons pu colliger 259 cas de malnutrition aigüe sévère sur 4724 enfants hospitalisés soit $5,5 \%$; dépendantes ainsi financièrement de leurs maris qui en majorité travaillaient dans le secteur informel $(81,08 \%)$ avec de faibles revenus. En effet, Steve Collins et al [19] ont également souligné le rôle de la pauvreté des parents dans la survenue de la malnutrition. Nous avons noté une prédominance féminine avec $52,51 \%$ soit un sex-ratio de 0,90 . Cette prédominance féminine est rapportée par Diarra N [7] au CSRéf de la commune I de Bamako et Diarra I [8] au CSRéf de Koutiala en 2014 qui ont observé respectivement 0,9 et 0,79 . Par contre, TRAORE $F$ [9] et SANGARE B [10] ont trouvé une prédominance masculine avec un sex-ratio de 1,26. De même l'enquête SMART 2014 [11] qui a trouvé un sex ratio égal à 1,2 . Cependant, II n'aurait pas d'influence du sexe sur la survenue de la malnutrition. L'âge moyen des patients était de 16,44 $\pm 11,21$ mois avec les extrêmes de 3 mois à 59 mois. La tranche d'âge 6 à 23 mois était la plus touchée par la malnutrition aigüe sévère avec $75,3 \%$. La vulnérabilité à la malnutrition de cette tranche d'âge a été constatée aussi par SOGOBA.H S [12], SISSOKO. F [13], et ZAKARI.K [14] qui ont obtenu respectivement $49,4 \% ; 53,2 \%$; et $31,1 \%$ des cas. Cela s'expliquerait peut-être par le rôle que joue l'alimentation de complément pour ces enfants. En effet à partir de 6 mois, le lait maternel n'est plus suffisant et l'alimentation de complément le plus souvent n'est pas introduite à temps ou n'est pas adaptée qualitativement et/ou quantitativement. L'allaitement exclusif a été observé chez $76,06 \%$ et $36,6 \%$ des patients n'avaient pas reçu d'aliments de supplément après 6 mois. Le marasme était la forme clinique de malnutrition la plus observée soit $87,64 \%$ contre $10,42 \%$ de kwashiorkor et $1,93 \%$ mixte.

Le niveau des indicateurs de performance à l'URENI

Dans notre série, $54,83 \%$ de nos patients étaient « traités avec succès ». Ce résultat est inférieur à ceux de SOGOBA.H S [10] à l'URENI du CSRéf de Diéma en 2009, de DIARRA N [7] au CSRéf de la commune I en 2014 et de la PCIMA [17] qui ont observé respectivement un taux de $83,2 \% ; 85,9 \%$ et de plus de $75 \%$. II est cependant supérieur à ceux de SISSOKO.F [13] à I'URENI Gabriel Toure en 2008 et de TRAORE F M [9] à I'URENI Gabriel Toure en 2014 qui ont trouvé 8,67\% et $45,68 \%$ respectivement.

Notre taux pourrait s'expliquer par l'association statistiquement significative qu'il y'avait entre les traités avec succès et la profession salariale des pères (OR : $2,700 ; p: 0,028) ;$ ou par la relation entre les traités avec succès et une prise en charge efficace du paludisme (OR: 2,564; $p: 0,035$ ). Le <<taux d'abandon>> du traitement nutritionnel était de $18,53 \%$ dans notre série. Ce taux est meilleur par rapport à ceux rapportés par SISSOKO.F [13] à I'URENI Gabriel Toure en 2008 et de TRAORE F M [9] à I'URENI Gabriel Toure en 2014 qui ont trouvé respectivement $67,6 \%$ et $34,2 \%$. Ce résultat est nettement supérieur à ceux de Diarra I [8] et de la PCIMA [17] qui étaient de $0,3 \%$ et de moins de $10 \%$ des cas respectivement.

Notre taux s'expliquerait par l'association statistiquement significative qu'il y'avait entre l'abandon et le poids 
financier (OR : 3,037; p: 0,005). Par rapport au « taux de décès ", il était de 23,55\%. Ce taux est supérieur à ceux de SISSOKO.F [13], de SAVADOGO.L [18], TRAORE F $M$ [19] et PCIMA [17] qui étaient respectivement $11,6 \%$, $16 \%, 9 \%$ et de moins de $15 \%$. Cela pourrait s'expliquer dans notre étude par l'association qu'il y'avait entre le taux de décès et les complications à l'admission (OR : 21,345 et $p: 0,001$ ). En effet, à l'admission déjà, en plus de l'échec au test de l'appétit, d'autres complications redoutables étaient déjà installées témoignant ainsi un retard de recours aux soins.

CONCLUSION

La malnutrition reste très fréquente au Mali. Sa prise en charge avec succès est possible s'il n'y a pas de complication associée. Les conditions socioéconomiques défavorables des parents (analphabétisme et bas revenu) et l'association des complications sont des facteurs qui influences considérablement les indicateurs de performance.

\section{Références}

1. OMS. La prise en charge de la malnutrition sévère. Manuel à l'usage des médecins et autre

Personnel de santé à des postes d'encadrement. Genève : OMS ; 2000.

2. Black RE, Victora CG, Walker SP. Maternal and child under nutrition and overweight in low-income and middleincome countries. Lancet 2013; 382: 427-451.

3. Robert E Black, Lindsay H Allen, Zulfiqar A Bhutta, global and regional exposures and health consequences. Lancet 2008; 371: 243-60.

4. OMS, I'UNICEF 2012. Améliorer la nutrition de l'enfant. Genève : UNICEF ; 2012 ; 132 :8.

5. Institut National de la Statistique et Direction Nationale de la Santé (Mali). Enquête Nutritionnelle Anthropométrique et de Mortalité rétrospective SMART $2015 ; 116: 44$

6. OMS. Prise en charge communautaire de la malnutrition aiguë sévère: Déclaration commune de l'Organisation mondiale de la Santé, du Programme alimentaire mondial, du Comité permanent de la nutrition du Système des Nations Unies et du Fonds des Nations Unies pour l'enfance. OMS ; $2007: 12$.

7. Diarra N. Les infections courantes dans le cas de la malnutrition aigüe sévère des enfants de 6 à 59 mois dans le service de pédiatrie du CSRéf de la commune I du district de Bamako. Thèse Med. Bamako 2014 :89.

8. Diarra I. Evaluation de la prise en charge de la malnutrition aigüe sévère chez les enfants de 6 à 59 mois à I'URENI du centre de santé de Référence de koutiala.[Thèse Med]. Bamako 2014 : 83.

9. Traore F. Aspects épidémio-clinique de la malnutrition aigüe sévère des enfants de 0 à 59 mois au CHUGT. Thèse Med. Bamako 2014 ; 77p Laura E Caulfield, and coll. Maternal and child nutrition:

10. Sangare B. Evaluation de la prise en charge de la malnutrition aigüe sévère dans le service de pédiatrie de l'hôpital de Gao. [Thèse Med]. Bamako 2009 :79.

11. Institut National de la statistique et Direction Nationale de la santé: Enquête Nutritionnelle Anthropométrique et de Mortalité rétrospective chez les enfants de 6 à 59 mois et des femmes de 15 à 49 ans SMART. Bamako $2015: 12$.

12. Sogoba $H$. Etude de la malnutrition aigüe sévère chez les enfants de 0 à 5 ans au centre de référence de DIEMA. [Thèse de Med] Kayes $2010: 103$.

13. SISSOKO F. Bilan d'activités de l'URENI des enfants malnutris sévères de 0 à 59 mois hospitalisés dans le service de pédiatrie du CHU GT. [Thèse Med] Bamako 2010 : 22-75-76

14. Zakari K. Etude des causes de "Non réponse » au traitement des malnutris sévères au

CRENI de l'hôpital national de Niamey chez les enfants de moins de 5 ans. [Thèse Med]

Bamako 2008

15. Coulibaly 0 . Connaissances, attitudes et pratiques des mères ou gardiennes d'enfants malnutris face à la malnutrition des enfants de 6 à 59 vus en consultation au CSRéf de Niono. [Thèse de Med] Ségou $2013: 86$.

16. Diop M. Evaluation de la prise en charge de la malnutrition aigüe sévère dans I'URENI du CSRéf de Markala. [Thèse de Med] Ségou 2014 : 70.

17. Ministère de la sante du Mali, Division nutrition/UNICEF. Protocole national révisé de la PCIMA et les normes de croissances. Bamako $2012: 6$.

18. Savadogo L, Zoetaba I et al. Prise en charge de la malnutrition aiguë sévère dans un centre de réhabilitation et d'éducation nutritionnelle urbain au Burkina-Faso. Revu Epidémio Santé Publique 2007; 55: 265-274.

19. Steve Collins, Nicky Dent, Paul Binns, Paluku Bahwere, Kate Sadler, Alistair Hallam. Management of severe acute malnutrition in children. Lancet $2006 ; 368$ : 1992-2000

\section{Liste des tableaux et figures :}

Tableau I: Répartition des enfants malnutris selon le mode d'alimentation, le motif de consultation et les signes physiques.

\begin{tabular}{lcc}
\hline Variables & $\mathbf{N = 2 5 9}$ & $\%$ \\
\hline Mode d'alimentation avant $\mathbf{6}$ mois & & \\
Allaitement maternel exclusif & 197 & 76,06 \\
Alimentation mixte & 44 & 16,66 \\
Allaitement Artificiel & 18 & 06,95 \\
Aliment de complément après 6 mois & & \\
Oui & 164 & 63,32 \\
Non & 95 & 36,68 \\
Motif de consultation & & \\
Anorexie & 173 & 66,79 \\
Toux & 172 & 66,41 \\
Diarrhée & 135 & 52,12 \\
Vomissement & 100 & 38,61 \\
Dyspnée & 9 & 3,47 \\
\hline
\end{tabular}




\begin{tabular}{lcc}
\hline Signes physiques & & \\
Fonte musculaire & 172 & 66,79 \\
Candidoses & 94 & 66,41 \\
Cheveux fins roux et cassants & 80 & 52,12 \\
Altération de l'état général & 65 & 38,61 \\
Pâleur & 36 & 03,47 \\
Lésions cutanées & 35 & 13,51 \\
OEdèmes des membres & 29 & 11,20 \\
inférieurs & & \\
Hépatomégalie & 29 & 11,20 \\
Distension abdominale & 18 & 06,95 \\
Convulsions & 15 & 05,79 \\
Coma & 04 & 01,54 \\
\hline
\end{tabular}

Tableau II : Répartition des patients selon les caractéristiques sociales des parents.

\begin{tabular}{|c|c|c|}
\hline $\begin{array}{l}\text { Caractéristiques sociales des } \\
\text { parents }\end{array}$ & $\mathrm{N}=259$ & $\%$ \\
\hline \multicolumn{3}{|l|}{ Niveau d'instruction des Pères } \\
\hline Ecole coranique & 14 & 05,41 \\
\hline Primaire & 22 & 08,49 \\
\hline Secondaire & 12 & 04,63 \\
\hline Aucun & 206 & 79,54 \\
\hline Inconnu & 05 & 01,93 \\
\hline \multicolumn{3}{|l|}{ Profession des pères } \\
\hline Fonctionnaire & 44 & 16,99 \\
\hline Secteur informel & 210 & 81,08 \\
\hline Inconnue & 05 & 01,93 \\
\hline \multicolumn{3}{|l|}{ Niveau d'instruction des Mères } \\
\hline Ecole coranique & 14 & 05,41 \\
\hline Primaire & 22 & 08,49 \\
\hline Secondaire & 12 & 04,63 \\
\hline Aucune instruction & 206 & 79,54 \\
\hline Non connue & 05 & 01,93 \\
\hline \multicolumn{3}{|l|}{ Profession des Mères } \\
\hline Commerçantes/vendeuses & 43 & 16,60 \\
\hline Elèves/étudiantes & 07 & 02,70 \\
\hline Femmes au foyer & 204 & 78,76 \\
\hline Inconnue & 05 & 01,93 \\
\hline
\end{tabular}




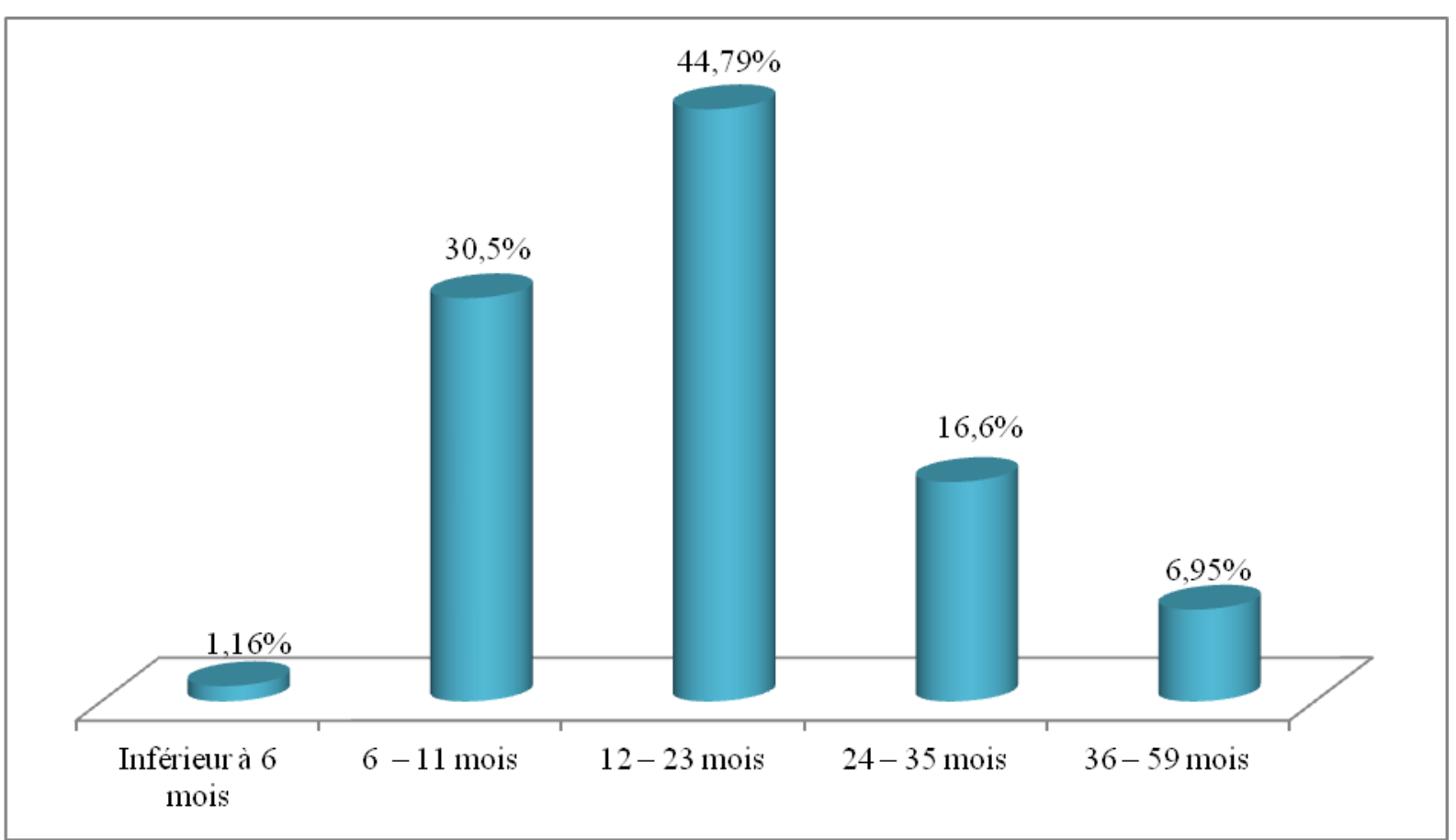

Figure 1 : Répartition des enfants malnutris selon la tranche d'âge.

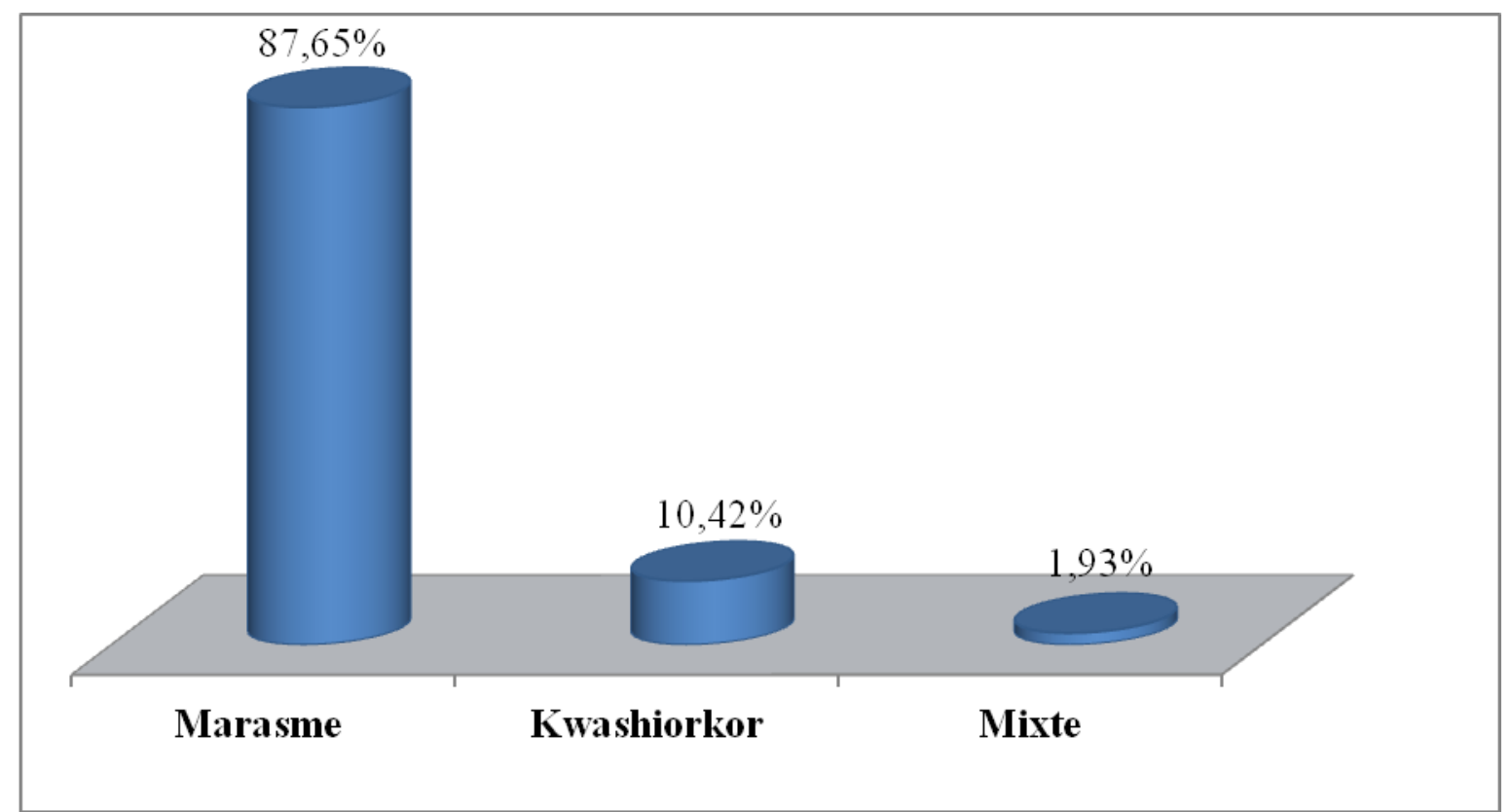

Figure 2 : Répartition des patients selon la forme de malnutrition. 


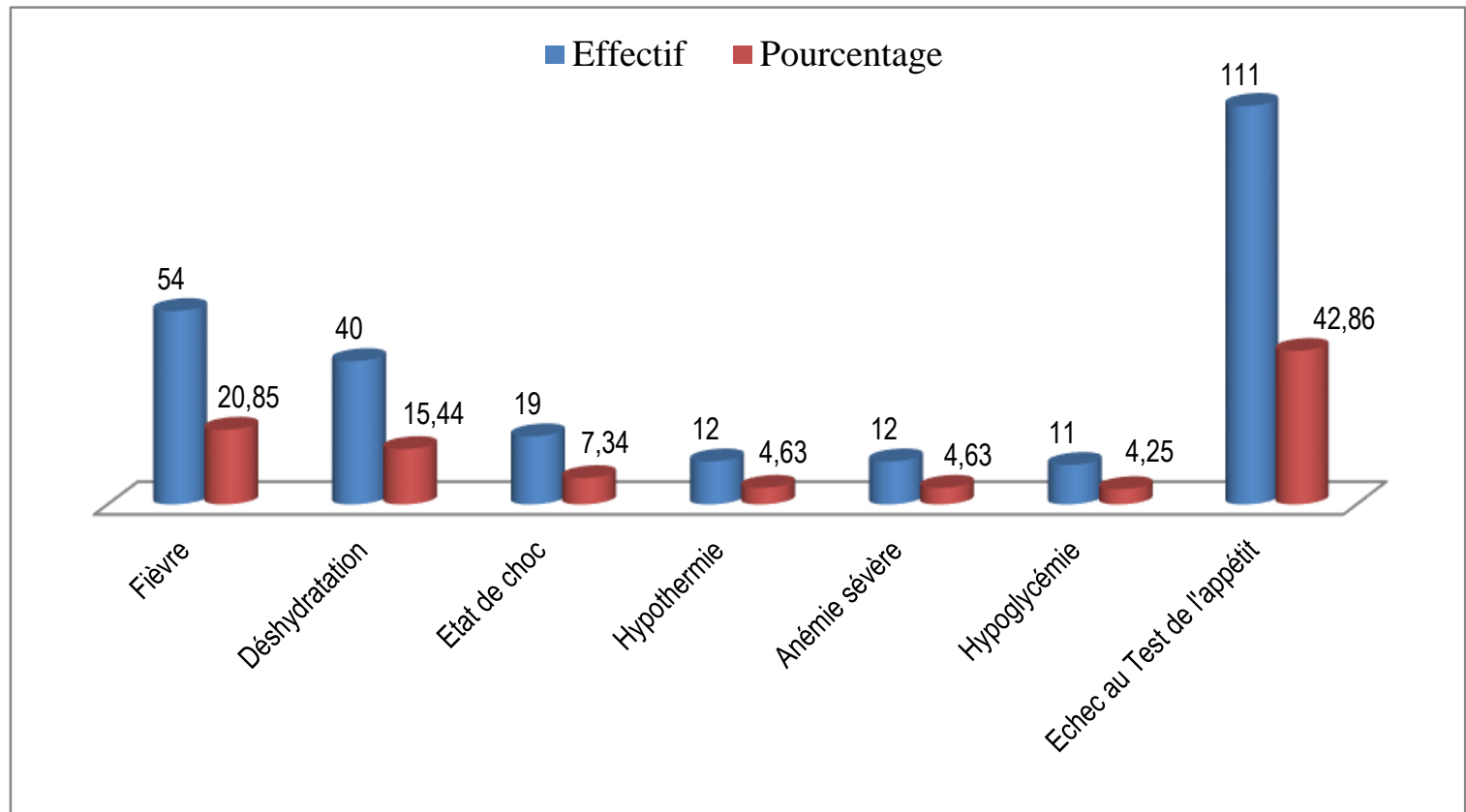

Figure 3 : Répartition des patients selon les complications à l'admission

Tableau III : Répartition des enfants malnutris selon le traitement.

\begin{tabular}{lll}
\hline Traitement & $\mathbf{N}=\mathbf{2 5 9}$ & $\%$ \\
\hline Molécules utilisées & & \\
Lait F75+F100+PPN & 226 & 87,26 \\
Lait F75+PPN & 30 & 11,58 \\
Lait F100 dilué & 3 & 1,15 \\
Durée de séjour & & \\
Moins de 4 semaines & 246 & 94,98 \\
$4-6$ semaines & 13 & 05,02 \\
Devenir & & \\
Traités avec succès & 142 & $54,83 \%$ \\
Décès & 61 & $23,55 \%$ \\
Abandon & 48 & $18,53 \%$ \\
Non répondant & 08 & $03,09 \%$ \\
\hline
\end{tabular}

Tableau IV : Facteurs influençant les indicateurs de performance à l'URENI.

\begin{tabular}{|c|c|c|c|c|c|c|}
\hline \multirow[t]{2}{*}{ Facteurs } & \multicolumn{2}{|c|}{ Traité avec succès } & \multicolumn{2}{|c|}{ Décès } & \multicolumn{2}{|c|}{ Abandon } \\
\hline & Odds Ratio & $\mathrm{p}$ & Odds Ratio & $p$ & Odds Ratio & $p$ \\
\hline \multicolumn{7}{|l|}{ Profession du père } \\
\hline Informel & Réf & & Réf & & Réf & \\
\hline Salarié & 2,7 & 0,028 & 0,472 & 0,179 & 0,583 & 0,376 \\
\hline \multicolumn{7}{|l|}{ Poids à l'admission } \\
\hline Moins de $5 \mathrm{Kg}$ & Réf & & Réf & & Réf & \\
\hline $5-7 \mathrm{Kg}$ & 1,553 & 0,211 & 1,162 & 0,738 & 0,52 & 0,114 \\
\hline Plus de7kg & 2,046 & 0,138 & 0,873 & 0,824 & 0,269 & 0,04 \\
\hline \multicolumn{7}{|l|}{ Pathologies Associées } \\
\hline Paludisme & 2,564 & 0,035 & 0,556 & 0,283 & 0,416 & 0,164 \\
\hline Gastroentérite & 2,807 & 0,058 & 0,457 & 0,224 & 0,737 & 0,652 \\
\hline Pneumopathie & 1,431 & 0,472 & 0,518 & 0,285 & 1,223 & 0,732 \\
\hline VIH & 1,341 & 0,519 & 0,785 & 0,715 & 0,811 & 0,699 \\
\hline Complications PCIMA à l'admission & 0,228 & 0,001 & 21,345 & 0,001 & 0,807 & 0,623 \\
\hline \multicolumn{7}{|l|}{ Contraintes financières } \\
\hline Problèmes financiers & 0,606 & 0,141 & 0,802 & 0,608 & 3,037 & 0,005 \\
\hline
\end{tabular}

